\title{
A new type of arthropod photoreceptor
}

\author{
Rafael Jordana $^{a}$, Enrique Baquero ${ }^{a}, *$, Luis M. Montuenga ${ }^{b}$ \\ ${ }^{a}$ Department of Zoology and Ecology, University of Navarra, PO Box 177, 31080 Pamplona, Navarra, Spain \\ ${ }^{\mathrm{b}}$ Department of Histology and Pathology, University of Navarra, PO Box 177, 31080 Pamplona, Navarra, Spain
}

Received 13 September 2000; accepted 23 January 2001

\begin{abstract}
A new type of photoreceptor for the phylum Arthropoda, found in the class Collembola (Arthropoda, Hexapoda) is reported. This new light-sensitive structure consists of a pair of interocular vesicles present in the genus Vesicephalus Richards, 1964 and is anatomically related to the cluster of ommatidia. The absence of a lens, the presence of a rabdome in the upper part of the vesicle and the reflection and refraction of light by a hemolymph bubble with incidence to the rhabdomeric structure are the main traits of this new photoreceptor. (C) 2001 Elsevier Science Ltd. All rights reserved.
\end{abstract}

Keywords: Arthropods morphology; New photoreceptor; New eye; Vesicephalus; Symphypleona; Collembola

\section{Introduction}

In Arthropoda, four types of photoreceptors have been classically considered: (1) the typical facetted eyes; (2) simple-lens eyes or frontal ocelli with cup-shaped retina; (3) median eyes, typical of larvae ('nauplius' eyes in Crustacea) which are classified as frontal eyes or frontal ocelli when they persist in adults; and (4) the frontal organs (originally paired) found in Collembola, most Crustacea and other Arthropoda. In Hexapoda, the nerve fibers arising from the latter end in special areas of the central protocerebrum called ocelli center (Marlier, 1941; Paulus, 1972b, 1979).

Since the early days of the study of photoreceptors in the class Collembola, two types were always described. A first type, usually named 'eyes', is comprised of two clusters of no more than eight ommatidia, found at each side of the head. The second type are ocelli that are found under the cuticle (Guthrie, 1906; Hesse, 1901) and are not apparent from the outside due to the absence of differentiations in the covering cuticle. These ocelli are located at different anatomical positions (Hesse, 1901; Marlier, 1941; Paulus 1972b, 1973): (1) single anterior frontal, described in detail in Orchesella, Entomobrya, Isotoma, Heteromurus, Podura, Sminthurus and Dicyrtoma; (2) single posterior dorsal, studied in Orchesella, Entomobrya, Isotoma, Sminthurus and Dicyrtoma; (3) lateral paired, found in Isotoma,

\footnotetext{
* Corresponding author. Tel.: +34-948-425600; fax +34-948-425649.

E-mail address: ebaquero@unav.es (E. Baquero).
}

Entomobrya and Tomocerus but not in Symphypleona; and (4) the ocelli found under the brain, i.e. deep inside the head cavity in Tomocerus (Marlier, 1941; Horridge, 1965; Barra, 1969). The exact distribution of these groups of photoreceptor cells, found in some cases under pigmented areas, and their relationship with the ocelli centre has been frequently studied (Marlier, 1941; Paulus, 1972b, 1973, 1979). The rhabdome of both the eyes and the ocelli has been described by means of light and electron microscopy (Barra, 1969; Paulus, 1972a,b, 1979). None of these studies describes any accessory structures in the cuticle nor under the rhabdome. Only in Podura (Paulus, 1972b) are the lateral ocelli situated just beneath the cuticle inside of a small nipple of $2 \mu \mathrm{m}$.

\section{Materials and methods}

Specimens of Vesicephalus europaeus Ardanaz and Pozo, 1985 were obtained using the Berlesse-Tullgren extraction method from litter in a beech forest (Fagus sylvatica) with Taxus baccata, Quercus robur and Corylus avellana. The understory of the forest mainly has Buxus sempervirens and soil covered by litter and moss. Due to very dense plant cover and its location within a narrow valley with no direct sunlight, the forest floor remains in shade throughout the year. The reported distribution of V. europeus is the beech forest of the northern part of the Iberian Peninsula.

The first specimens captured were fixed and processed for scanning electron microscopy (SEM). Fixative consisted of a mixture of methanol, acetic acid and water 2:2:1 (Saito 

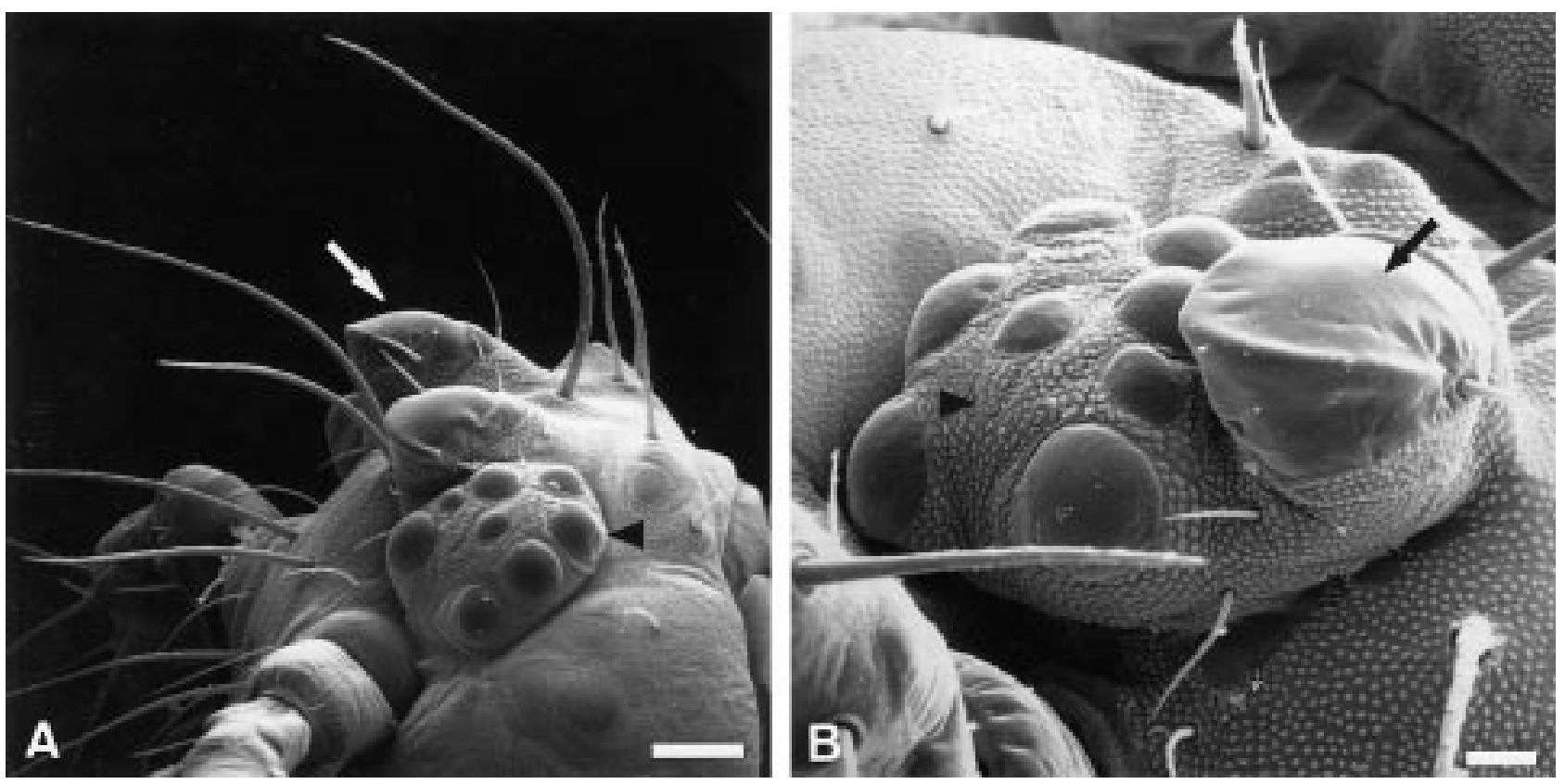

Fig. 1. Electron micrographs of V. europaeus. (A) Eye (arrowhead) and interocular vesicle (arrow) in a close up lateral view by SEM (bar $50 \mu m$ ). (B) Same as (A) in frontal view showing the close relation between eye and vesicle (bar $20 \mu \mathrm{m}$ ).

and Osakabe, 1992). After a washing step, samples were dehydrated through a series of increasing concentrations of ethanol up to $100 \%$. Complete desiccation was achieved by the $\mathrm{CO}_{2}$ critical point technique. Samples were then covered by a $16 \mathrm{~nm}$ thin layer of molecular gold using the Emitech K550 equipment. The observation of the head of the specimens by SEM (Zeiss 940 A) [Fig. 1(A)] prompted us to collect new specimens to perform other morphological techniques, such as transmission electron microscopy (TEM) or conventional light microscopy of stained sections. Part of the new collected specimens were processed for TEM. Dissected heads were fixed in $4 \%$ glutaraldehyde in cacodylate buffer for $24 \mathrm{~h}$, postfixed in $1 \%$ osmium tetroxide, dehydrated through propylene alcohol and embedded in Epon. Other specimens were embedded in paraffin for light microscopy studies after $24 \mathrm{~h}$ fixation with either Bouin's fluid or $10 \%$ formalin in insect hemolymph isotonic buffer.

\section{Observations and discussion}

The genus Vesicephalus includes four species, one European and three American. Its distinctive feature, from which the name is taken, is a pair of vesicles found between the eye clusters. The observation of living specimens under the dissecting microscope allowed us to visualise and video record the paired head vesicles. Under certain illumination, these vesicles appear translucent and bright in living animals [Fig. 2(C)]. This observation led us to hypothesise a possible photoreceptor function. Our suggestion was strongly supported by a SEM frontal view of the head that showed a very clear anatomical relationship of these vesicles with the eight corneolae found in the eye cluster [Fig. 1(B)]. As has been mentioned in the Introduction, the 'eyes' of Collembola have no more than eight corneolae and thus, this vesicular structure appeared as a very unique element if a photoreceptor role was shown. SEM also showed that the dorsal surface of the vesicle is very smooth [Fig. 1(B)], in contrast to the characteristic reticular pattern of the cuticle of the rest of the body. Histological studies were then carried out in order to determine whether the cells comprising the vesicle displayed a rhabdome-like structure that is diagnostic of a photoreceptor function. The heads of the specimens for regular histological studies were sectioned along two planes, transversal [Fig. 2(A)] and longitudinal [Fig. 2(B)], and a rhabdome-like structure was clearly observed [Figs. 2(B) and (D)]. The smooth external surface of the vesicle is about $86 \times 66$ microns in area and has a complex histological organisation comprising several cellular and acellular structures. The external cuticular surface of the dorsal and frontal side of the vesicle is smooth and the cells below the cuticle lack any pigment. There are five traits that allow us to define the vesicular structure of the genus Vesicephalus that we describe here as a new type of photoreceptor that has not been previously described in Arthropoda.

1. The presence of a cuticular projection (so far called vesicle) with a very smooth surface and lack of pigmentation [Fig. 1(A) and 2(C)] which allows the passage of light. In contrast, there is a thin, pigmented layer surrounding the outer limits of the vesicle below the rough and reticulated part of the cuticle. A similar but more prominent pigment layer isolates each one of the 

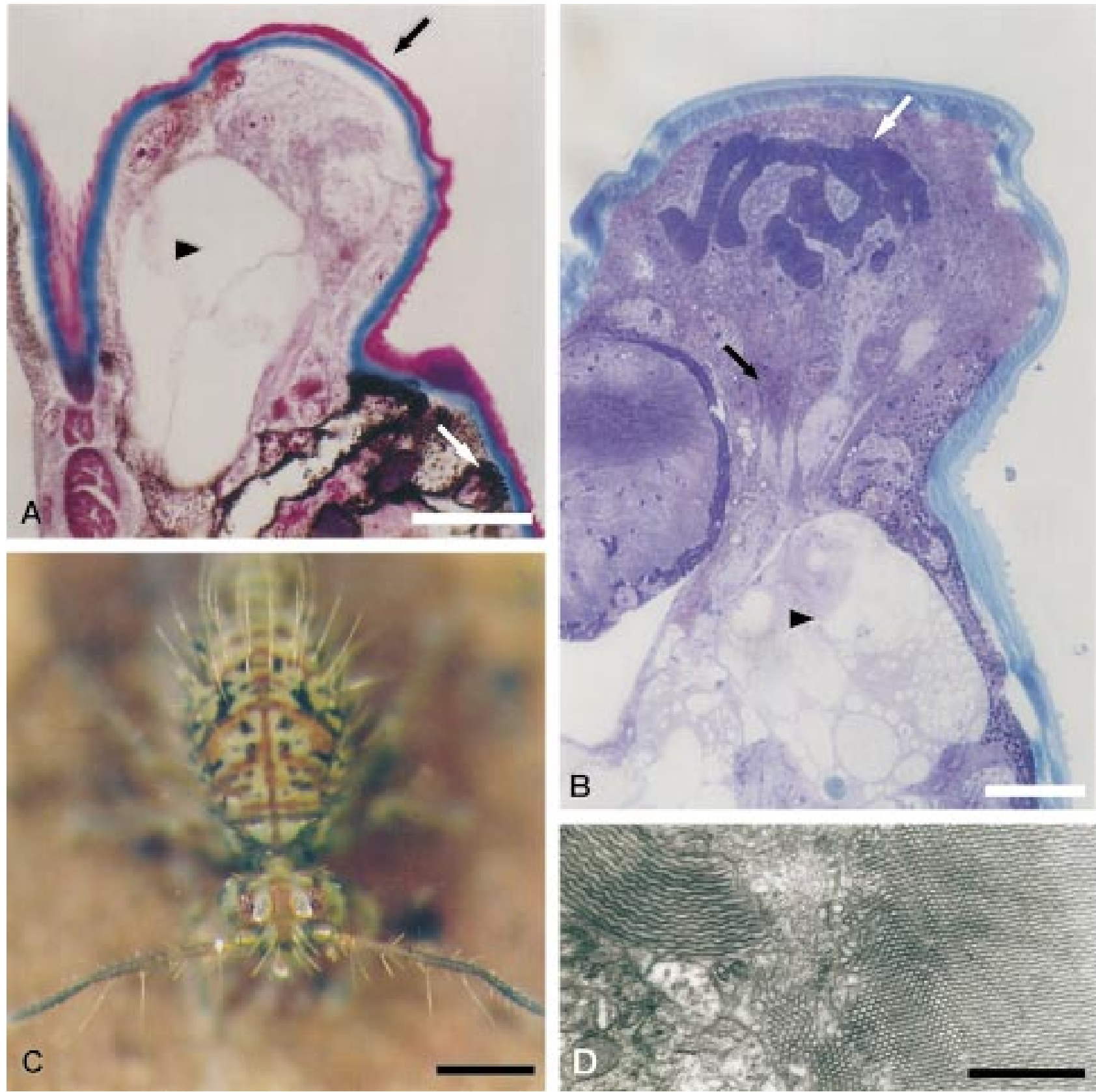

Fig. 2. Light and electron micrographs of V. europaeus. (A) Transversal section of the head of V. europaeus at eye and vesicle level in a paraffin embedded specimen stained with Masson's trichrome: vesicle (black arrow), one of the ommatidia of the eye (white arrow) and hemolymphatic space (arrowhead) (bar $40 \mu \mathrm{m}$ ). (B) Longitudinal section of the head at vesicle level in a plastic embedded specimen stained with toluidine blue. The apical rhabdome is seen as a dense convoluted membranous structure (white arrow). The arrowhead points to the hemolymphatic space and the black arrow to the nervous cells (bar $20 \mu \mathrm{m}$ ). (C) Dorsal view of a live specimen of Vesicephalus europaeus clearly showing the bright interocular vesicles (bar $400 \mu \mathrm{m}$ ). (D) TEM (transmission electron microscopy) detail of the rhabdomeric tubules showing different orientations (bar $0.1 \mu \mathrm{m}$ ).

ommatidia of the compound eye. The total external surface of the vesicle is approximately equivalent to the sum of the external surfaces of the eight corneolae of the 'eye', i.e. $9100 \mu \mathrm{m}^{2}$ for the vesicle and $12,800 \mu^{2}$ for the sum of the corneolae [Fig. 1(B)].

2. There is no lens. The light seems to reach the rabdome without any structure acting as a lens. Light can enter the photoreceptor from two different zones, dorsal and anterior, which are separated by a slightly thicker cuticular fold. This fold is not an artefact as has been observed in every specimen of Vesicephalus europaeus Ardanaz and Pozo, 1986 studied by us with SEM or TEM and also in the paratype of Vesicephalus crossleyi Snider, 1985 kindly provided by Dr Snider for our SEM studies.

3. In the apical region of the vesicle, below the cuticle, a group of no more than ten photosensitive cells with their 


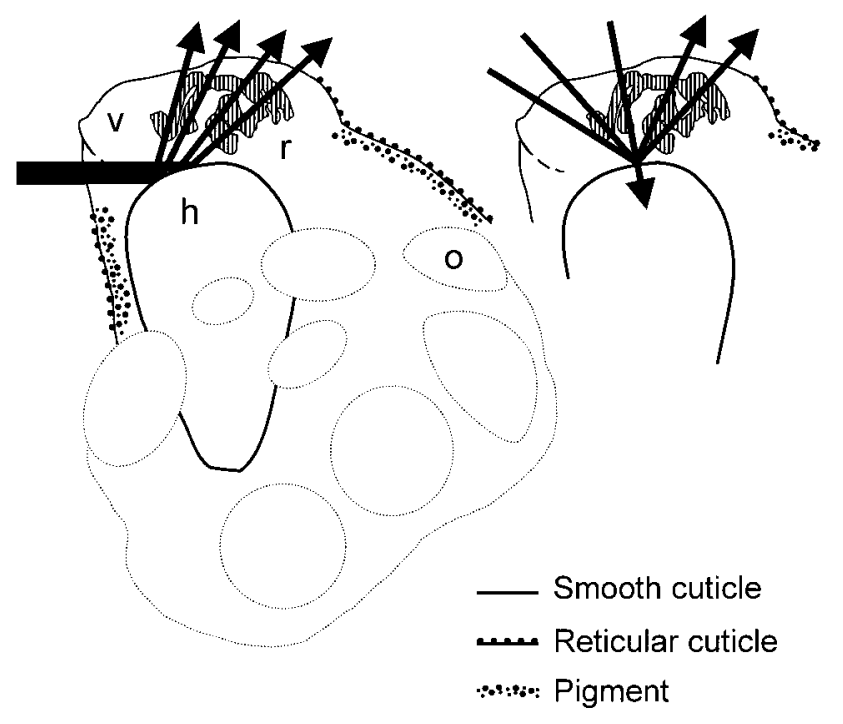

Fig. 3. Lateral view of the ocular area of Vesicephalus europaeus to show light direction and the probable pathway through frontal and dorsal parts of the vesicle ( $\mathrm{v}$ : vesicle, h: hemolymphatic bubble, r: rhabdome, o: ommatidium).

nuclei in the periphery, surround a dense irregular and convoluted membranous structure [Fig. 2(B)], that under the TEM shows the clear tubular pattern of a rabdome [Fig. 2(D)]. This structure is very similar to what has been described in the frontal organs of Collembola (Barra, 1969; Paulus, 1972b). The rhabdome is located in the posterior region of the vesicle. Very likely, it receives direct light from the exterior and also possibly reflected light from the interphase tissue/hemolymph bubble that lies below [Fig. 2(A)]. This reflexion effect is clearly visible in the living animal as is shown in Fig. 2(C).

4. The rhabdomeric cells are continuous with a thin nerve fibre that joins the neural tract that leaves the eight corneolae of the eye before it connects with the anterior lateral brain. It is possible that this rhabdomeric nerve could be homologous to the lateral ocelli nerves found in other species of Collembola. These lateral ocelli nerves arise from the ocellar centre and join the ocular nerve.

5. Below the rhabdomeric cells an apparently hollow space is found [Figs. 2(A) and (B)]. This 'bubble' is continuous with the hemolymphatic space and thus is filled by hemolymph as can be observed with the optical microscope in serial longitudinal and cross sections of the head (data not shown). This is the most likely cause of the bright appearance of the vesicle when the living animal is exposed to an intense light source [Fig. 2(C)]. This is a similar effect as observed when a tapetum lucidum is present in the eye of other animals like spiders (although it is not a bona fide tapetum lucidum because it lacks the typical multilayered reflectant cell structure).

In summary, the anatomical and histological characteristics of the vesicle present in Vesicephalus suggest that it is a visual organ with some degree of complexity, never before described in Collembola or any Arthropoda (Paulus, 1972b; Autrum, 1979, 1981; Harrison, 1991).

At present, Collembola are considered as a class within the superclass Hexapoda. The morphology of the photoreceptors within this group is quite different as compared to that of true insects. Insects have both multifaceted compound eyes and ocelli with simple lens (a pair and a single one at most). As has been mentioned before, Collembola have 'eyes' and simple ocelli. The vesicular structure that we describe in the present study, cannot be considered as one ommatidium more of the group that comprise the collembolan 'eye', as it does not have the typical structure of an ommatidium. It has indeed some relationship with them as it is present in the same cuticular prominent plane of the head [Fig. 1(B)]. It cannot be identified either with the ocelli present in Collembola, as the ocelli do not have cuticular modifications or accessory structures such as the hemolymph bubble. Several studies have focused on these ocelli of Collembola. It has been suggested that an hypothetical ancestor could have had up to eight pairs of innervated ocelli, with all nerve fibres joining in the ocellar centre of the protecerebrum. In Crustacea and Collembola there is a trend to fuse paired ocelli into single structures. In Collembola, paired (lateral) ocelli are only found in four genera: Podura, Isotoma, Entomobrya and Tomocerus (Marlier, 1941; Paulus, 1972b). In Podura lateral ocelli have been described closely located to the ommatidia situated just beneath the cuticle inside of a small nipple of $2 \mu \mathrm{m}$ and $16 \mu \mathrm{m}^{2}$ of surface, with reticulated cuticle, very diferent from the structure described in this paper. They are innervated from the same ocellar centre than frontal and dorsal ocelli.

In conclusion, until other genera of the class Collembola are studied, this is the first description of a photoreceptor with some degree of anatomical differentiation from the already known 'eye' comprised of eight ommatidia. This new structure could be originally related to the lateral ocelli of Collembola.

The possible function of this new photoreceptor may be related to detection of sudden changes in lighting, such as occasioned by an approaching predator. The size, the reflection and refraction of light by the hemolymph bubble and its double incidence to the rhabdomeric membranes is very likely a mechanism to increase the light sensitivity of this photoreceptor. The absence of a lens and the presence of a rhabdome in the upper part of the vesicle are incompatible with the formation of images and thus the structure may be useful only as a light intensity sensor. A hypothetical functional scheme of the light pathway through this photoreceptor is provided in Fig. 3.

\section{Acknowledgements}

We are especially grateful to Carlos García-Vivanco who 
helped us in the sample collection and to Blanca Irigoyen and Isabel Ordoqui (Department of Histology and Pathology, University of Navarra) for their contributions in the processing for histological studies and to Dr Mancini (Department of Physics in the University of Navarra) for his assessment of optical function of this photoreceptor. Also to Dr Snider (Michigan State University) for the loan of a paratype of Vesicephalus crossleyi.

\section{References}

Autrum, H., 1979, 1981. Handbook of Sensory Physiology. VII/6a. Springer-Verlag, Berlin, Heidelberg \& New York.

Barra, J.A., 1969. Les photorécepteurs des Collemboles. Nouvelles formations à structure 'rhabdomérique', propres au genre Tomocerus (Insectes, Collemboles). CR Acad. Sci. 268, 2088-2090.

Guthrie, J.E., 1906. Studies of the Collembolan eye. Iowa Acad. Sci. 13, 239-243 Plate XVIII.

Harrison, F.W., 1991. Microscopic Anatomy of Invertebrates. Willey-Liss, New York.

Hesse, R., 1901. Untersuchungen über die Organe der Lichtempfindung bei niederen Thieren VIII. Von den Arthropoden-Augen. Zeitschr. wiss. Zool. 70, 347-473.

Horridge, G.A., 1965. Arthropoda: receptors for light and optic lobe. In: Bullock, T.H., Horridge, G. (Eds.). Structure and Function in the Nervous System of Invertebrates, Vol. II. W.H. Freeman Company, San Francisco-London, pp. 1097-1111.

Marlier, G., 1941. Recherches sur les organes photorécepteurs des insectes Aptilotes. Ann. Soc. Roy. Zool. Belg. 72, 204-236.

Paulus, H.F., 1972a. Zum Feinbau der Komplexaugen einiger Collembolen Eine vergleichend-anatomische Untersuchung (Insecta, Apterygota). Zool. Jb. Anat. 89, 1-116.

Paulus, H.F., 1972b. Die Feinstruktur der Stirnaugen einiger Collembolen (Insecta, Entognatha) und ihre Bedeutung für die Stammesgeschichte der Insekten. Z. Zool. Syst. Evolut.-forsch. 10, 81-122.

Paulus, H.F., 1973. Die Feinstruktur der Stirnaugen einiger Collembolen (Insecta, Entognatha) und ihre Bedeutung für die Stammesgeschichte der Mandibulaten. Verh. Dtch. Zool. Ges. 66, 56-60.

Paulus, H.F., 1979. Eye structure and the monophyly of the arthropoda. In: Gupta, A.P. (Ed.). Arthropod Phylogeny. Van Nostrand Reinhold Company, pp. 299-383 762 pp.

Saito, Y., Osakabe, M.H., 1992. A new fixation method for preparing mite specimens for optical and SEM microscopic observations. Appl. Entomol. Zool. 27 (3), 427-436. 